\title{
Does depression affect memory in older adults?
}

\section{“...depression may be a causal risk factor for dementia, a prodromal sign, a reaction to early cognitive deficits or a symptom of the dementia itself."}

The lifetime risk of major depression in the general population ranges between 10 and $15 \%$ [1]. A number of studies have now demonstrated that depression risk is somewhat lower in elderly populations [2]. This, combined with its association to cognitive problems and dementia, suggests that depression in older adults must be taken very seriously. Formally defined by the Diagnostic and Statistical Manual of Mental Disorders (DSM-IV) [1], a major depressive episode is defined according to several criteria. First, diagnosed individuals must exhibit at least five depression symptoms, one of which must be mood disturbance or anhedonia. Second, the symptoms must not meet the criteria for a mixed episode of mood disturbance involving manic symptoms. Third, the symptoms must cause clinically significant distress or impairment. Fourth, they cannot be due to the effects of a substance or general medical condition. Finally, the symptoms must not be better accounted for by bereavement.

One limitation of the categorical approach is that different individuals may achieve a diagnosis by endorsing very different clusters of symptoms. This is important because different symptoms appear to have different consequences for functional impairment as well as response to treatment [3]. In addition, there is no clear evidence that elderly individuals who meet the criteria for four depression symptoms are qualitatively different from those who meet the criteria for five or more [2].

Although categorical models have been helpful in operationalizing depression criteria and guiding research, there is increasing evidence that depression in the general population is most likely to be dimensional rather than categorical in nature. Recent work has supported the dimensional nature of depression in the elderly as well, with studies showing that different depression symptoms are associated with different types and levels of impairment [2]. Similarly, subthreshold depressive symptoms have been demonstrated to be important in the elderly and are associated with cognitive dysfunction, cognitive decline and decreased regional brain volume [4].

Cognitive problems in the elderly have been conceptualized in a variety of ways ranging from mild cognitive impairment (MCI) to dementia. MCI describes elderly individuals who have mild memory or cognitive impairment that is not associated with significant disability and cannot be attributed to any psychiatric or medical condition [5]. The term 'cognitive impairment no dementia' (CIND) is also commonly used to describe such mild cognitive conditions in the elderly, but it does not exclude those with affective problems [6]. Although the risk of subsequent dementia diagnosis is substantially elevated in both MCI and CIND groups, relative to the general population, outcomes are heterogeneous. A high proportion of individuals eventually progress to dementia, but it is also not uncommon for individuals to return to normal cognitive functioning at follow-up. One factor accounting for improvement may be remission of depression. Gallasi and colleagues demonstrated significant improvements in anterograde memory performance in a group of elderly individuals with major depression over a 6-month period of antidepressant treatment [7]. However, this study revealed that the remitted major depression patients still displayed relatively worse memory performance compared with a matched healthy control group. Similar findings have emerged from other studies examining cognitive functioning after remission or the successful treatment of late-life depression (LLD) [8].

More severe memory and cognitive problems that meet the criteria for dementia have also been demonstrated to be associated with depression. However, findings are mixed concerning the relationship between depressive symptoms and dementia in the elderly. Existing work suggests that depression may be a causal risk factor for dementia [8], a prodromal sign [6], a reaction to early cognitive deficits or a symptom of the
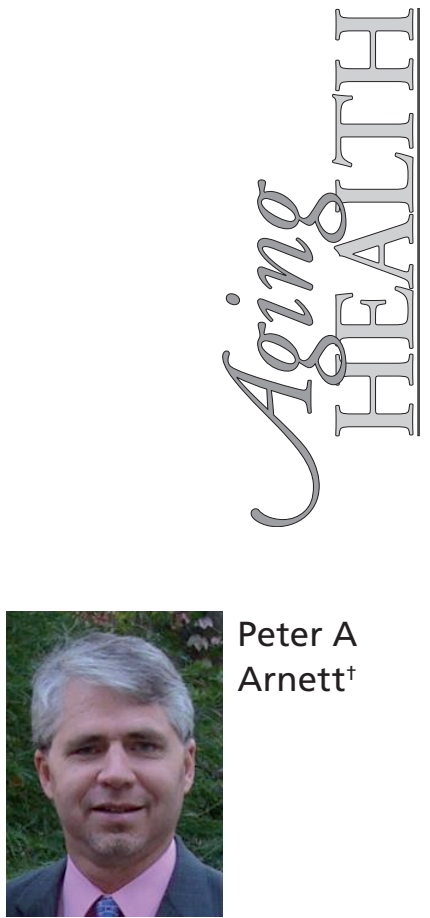

Peter A Arnett $^{\dagger}$

${ }^{+}$Author for correspondence Pennsylvania State University, Psychology Department, 522 Bruce V. Moore Bldg, University Park, PA 16802-3104, USA

Tel.: +1 8148631733

Fax: +18148631331 paa6@psu.edu

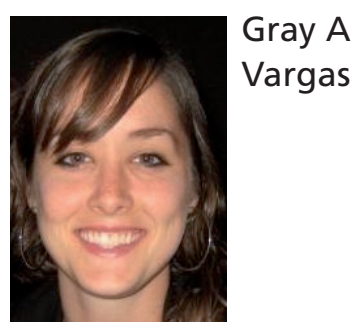

future medicine $^{\text {pisg }}$ 
dementia itself [9]. Part of the confusion comes from the high comorbidity between the two disorders: cognitive deficits are very common among older individuals with LLD and depression is very common in those with dementia. One study found that $34 \%$ of elderly individuals with current or past depression had CIND [6]. Furthermore, CIND often progresses to dementia, with studies typically showing that approximately 20-35\% of individuals with CIND convert to dementia after follow-up periods ranging from 2 to 5 years [10,11]. Similarly, subjective memory complaints (SMC) are likely to be the main concern expressed by those with LLD, and such complaints have been found to be more related to depression than objectively measured current cognitive functioning $[12,13]$. Conversely, $20-30 \%$ of Alzheimer's disease (AD) patients have been demonstrated to have significant depressive symptoms [9].

"With ... Alzheimer's disease and
depression having similar detrimental
effects on similar neural structures, it
is clear how their presentation can
lead to diagnostic confusion and
how one might increase the risk of
... the other."

One theory to explain how depression might serve as a causal risk factor for more severe memory and cognitive problems, such as those that manifest in $\mathrm{AD}$, involves hypothalamic-pituitary-adrenal (HPA) axis functioning. Depression involves chronically elevated levels of glucocorticoids, especially in the elderly, which is thought to lead to hippocampal cell injury or death and a decrease in neurogenesis in the dentate gyrus [8]. In fact, some studies have shown that individuals with LLD have reduced hippocampal volume, and this volume correlates with duration of lifetime depression. It is hypothesized that these cellular mechanisms increase the hippocampus' vulnerability to damage through other pathophysiologic mechanisms such as AD [8]. Some studies have found that depression doubled an individual's risk of developing AD or other types of dementia, with risk correlating with the number of previous depressive episodes. In addition, $\mathrm{AD}$ patients with current or past depression have been demonstrated to have more hippocampal amyloid plaques and neurofibrillary tangles than those with no history of depression. With both $\mathrm{AD}$ and depression having similar detrimental effects on similar neural structures, it is clear how their presentation can lead to diagnostic confusion and how one might increase the risk of developing the other.

\section{"Subjective memory complaints have been found to be more strongly related to current depression and personality traits ... than with objectively measured current cognitive functioning or white matter lesions."}

Another possible explanation for how depression might lead to cognitive deficits in the elderly is via its effects on processing speed. Sheline and colleagues found that processing speed mediated 14 out of the 16 relationships between predictor variables (such as demographic and health risk factors) and cognitive functioning in patients with LLD [14]. Executive functioning was also an important factor, mediating 11 out of 16 relationships. Although depression severity was equally related to all cognitive domains, it only made a significant independent contribution to working memory. Furthermore, after controlling for processing speed, depression severity no longer predicted performance of working memory, episodic memory, language or executive functioning. Other studies have found that there might also be a primary episodic memory deficit in patients with LLD [15]. Regarding the possible substrate of these difficulties, it has been postulated that vascular risk factors, especially white matter changes in the frontostriatal pathways, could be responsible for slowed processing speed and executive dysfunction in older individuals [16]. Alternatively, depression may directly affect cognitive performance by lowering motivation and self-esteem [13].

Clinically, older individuals, especially those with LLD, are likely to present with SMCs as their chief concern. However, care must be taken when using such complaints as an indicator of current or future dementia. SMCs have been found to be more strongly related to current depression and personality traits, such as neuroticism, than with objectively measured current cognitive functioning or white matter lesions [13]. It remains unclear whether individuals also need to have baseline cognitive impairment in order for the SMCs to be predictive of further cognitive decline. Since some definitions of MCI exclude depression and other psychiatric disorders that 
contribute to cognitive impairment, a diagnosis of CIND is useful for individuals with comorbid depression [6].

Several biological variables have been studied in relation to cognition in the elderly population, including activation in the cingulate cortex and of the 5-HTT allele. Elderly patients with subclinical depressive symptoms have been demonstrated to have lower activity in the dorsal posterior cingulate cortex [17]. In nondepressed elderly individuals, one study found an interaction between waking cortisol levels and the 5-HTT's' allele, such that those with smaller hippocampi and higher cortisol levels only had worse delayed recall if they carried the 's' allele [18]. It was concluded that the negative effect of the 's' allele on memory was mediated by the HPA axis.

\section{Conclusion}

Does depression affect memory in older adults? This editorial suggests that the answer is: 'yes, but...' There is clear evidence that treatment of LLD results in improved memory and other cognitive functions. This suggests that depression causally affects memory in older adults. With that said, most studies demonstrate that the improvement in cognitive functioning that depressed individuals show does not bring them up to a level that is comparable with healthy controls. Such findings suggest alternative causal possibilities. The decreased processing speed associated with depression also appears to play a role in the memory problems experienced by older adults with depression [14]. Studies on more severe manifestations of memory problems, such as those found in $\mathrm{AD}$, suggest that depression may serve as a risk factor for AD. Butters and colleagues presented an appealing theory suggesting that depression across the lifespan may cause increased vulnerability to AD by increasing glucocorticoids, which can lead to hippocampal cell injury or death and that, in turn, may lead to hippocampal atrophy and, ultimately, AD [8]. Subjective memory complaints in depressed older adults have a weak correlation with objective memory problems and are most associated with current depression. Thus, such subjective complaints cannot be relied upon clinically and need to be verified by objective neurocognitive testing. In the final analysis, depression and memory complaints in older adults must be taken very seriously. Treatment of depression may lead to improved memory functioning, but the very presence of depression in an older adult has implications for processing speed, memory and other cognitive functions. In addition, depression may represent a marker for more serious memory problems that may evolve into AD or other types of dementia.

"There is clear evidence that
treatment of late-life depression
results in improved memory and
other cognitive functions."

\section{Financial \& competing interests disclosure}

The authors have no relevant affliations or financial involvement with any organization or entity with a financial interest in or financial conflict with the subject matter or materials discussed in the manuscript. This includes employment, consultancies, honoraria, stock ownership or options, expert testimony, grants or patents received or pending, or royalties.

No writing assistance was utilized in the production of this manuscript.

\section{Bibliography}

1. American Psychiatric Association: Diagnostic and Statistical Manual of Mental Disorders (4th Edition). American Psychiatric Association, Washington, DC, USA (1994).

2. Anderson TM, Slade T, Andrews G, Sachdev PS: DSM-IV major depressive episode in the elderly: the relationship between the number and the type of depressive symptoms and impairment. J. Affect. Disord. 117, 55-62 (2009).

3. Andrews G, Brugha T, Thase ME, Duffy FF, Rucci P, Slade T: Dimensionality and the category of major depressive episode. Int. J. Methods Psychiatr. Res. 16, S41-S51 (2007).
4. Dotson VM, Beason-Held L, Kraut MA, Resnick SM: Longitudinal study of chronic depressive symptoms and regional cerebral blood flow in older men and women. Int. J. Geriatr. Psychiatry 24, 809-819 (2009).

5. Panza F, Frisardi V, Capurso C et al.: Possible predictors of vascular cognitive impairment no dementia. J. Am. Geriatr. Soc. 57, 943-944 (2009).

6. Steffens DC, McQuoid DR, Potter GG: Outcomes of older cognitively impaired individuals with current and past depression in the NCODE study. J. Geriatr. Psychiatry Neurol. 22, 52-61 (2009).
7. Gallassi R, Di Sarro R, Morreale A, Amore M: Memory impairment in patients with late-onset major depression: the effect of antidepressant therapy. J. Affect. Disord. 91, 243-250 (2006).

8. Butters MA, Young JB, Lopez O: Pathways linking late-life depression to persistent cognitive impairment and dementia. Dialogues Clin. Neurosci. 10, 345-357 (2008).

9. Tsuno N, Homma A: What is the association between depression and Alzheimer's disease? Expert Rev. Neurother. 9, 1667-1676 (2009).

10. Hsiung GY, Donald A, Grand J et al.: Outcomes of cognitively impaired not demented at 2 years in the Canadian cohort study of cognitive impairment and related dementias. Dement. Geriatr. Cogn. Disord. 22, 413-420 (2006). 
11. Palmer K, Wang HX, Bäckman L, Winblad B, Fratiglioni L: Differential evolution of cognitive impairment in nondemented older persons: results from the Kungsholmen project. Am. J. Psychiatry 159, 436-442 (2002).

12. Minett TS, Dean JL, Firbank M, English P, O'Brien JT: Subjective memory complaints, white-matter lesions, depressive symptoms, and cognition in elderly patients. Am. J. Geriatr. Psychiatry 13, 665-671 (2005).

13. Reid LM, MacLullich AMJ: Subjective memory complaints and cognitive impairment in older people. Dement. Geriatr. Cogn. Disord. 22, 471-485 (2006).

14. Sheline YI, Barch DM, Garcia K et al.: Cognitive function in late life depression: relationships to depression severity, cerebrovascular risk factors and processing speed. Biol. Psychiatry 60, 58-65 (2006).
15. Delaloye C, Baudois S, de Bilbao F et al.: Cognitive impairment in late-onset depression: limited to a decrement in information processing resources? Eur. Neurol. 60, 149-154 (2008).

16. Taylor WD, MacFall JR, Payne ME et al:: Late-life depression and microstructural abnormalities in dorsolateral prefrontal cortex white matter. Am. J. Psychiatry 161, 1293-1296 (2004).

17. Woo SL, Prince SE, Petrella JR, Hellegers C, Doraiswamy PM: Modulation of a human memory circuit by subsyndromal depression in late life: a functional magnetic resonance imaging study. Am. J. Geriatr. Psychiatry 17, 24-29 (2009).

18. O’Hara R, Schröder CM, Mahadevan R et al: Serotonin transporter polymorphism, memory, and hippocampal volume in the elderly: association and interaction with cortisol. Mol. Psychiatry 12, 544-555 (2007).

\section{Affiliations}

- Peter A Arnett

Pennsylvania State University, Psychology Department, 522 Bruce V. Moore Bldg, University Park, PA 16802-3104, USA Tel.: +1 8148631733 Fax: +1 8148631331 paa6@psu.edu

- Gray A Vargas

Pennsylvania State University, Psychology Department, 522 Bruce V. Moore Bldg, University Park, PA 16802-3104, USA

Tel.: + 18148631733

Fax: +1 8148631331

grayvargas@gmail.com 